

\section{Sumário}

Dossiê Temático: Direito Internacional Econômico.........................................................1

EDITORIAL:

Resultados da III Conferência Bienal da Red DEI ................................................. 3

Michelle Ratton Sanchez-Badin (em nome da Diretoria da Red DEI), Fabio Costa Morosini e Lucas da Silva Tasquetto (em nome dos organizadores da III Conferência da Red DEI)

Direito Internacional Econômico no Brasil: QUem Somos E o QUE faZemos? EvidênCIas EMPÍRICAS DE 1994 A 2014

Michelle Ratton Sanchez Badin, Fabio Costa Morosini e Inaê Siqueira de Oliveira

UM ESPAÇO PARA PENSAR EM ALTERNATIVAS? A ACADEMIA LATINO-AMERICANA DE DIREITO INTERNACIONAL ECONÔMICO FRENTE À ORDEM ECONÔMICA GLOBAL

Nicolás Marcelo Perrone

Grupo de alto Nível Brasil - Urugua (GAN): um Novo paradigma para a INTEgraÇão PRODUTIVA NO MERCOSUL

Alebe Linhares Mesquita e Vivian Daniele Rocha Gabriel

O COMÉRCIO de SERVIÇOS ENTRE Brasil E URUGUAI: LiberalizaÇÃo, DESAFIOS E PERSPECTIVAS DO SETOR DE TECNOLOGIA DA INFORMAÇÃO E COMUNICAÇÃO (TIC) E SOFTWARES

Vivian Daniele Rocha Gabriel e Alebe Linhares Mesquita

Core Labor Standards No Regime de PREFERÊNCIAS TARIFÁrias No MERCOSUL: A NeCESSIDADE DE HUMANIZAÇÃO DO COMÉRCIO INTERNACIONAL

Martinho Martins Botelho e Marco Antônio César Villatore

ACordo TRIMS: FleXibilizaÇão ou Não? Política de CONTEÚdo loCal, PROCESSO PROdUTIVO BÁSICO (PPB) E OS DESAFIOS PARA A INDÚSTRIA BRASILEIRA E A INTEGRAÇÃo LATINO-AMERICANA 100

Natália Figueiredo 
ESTUDIOS DE CASO DE RECHAZOS EN FRONTERA DE EXPORTACIONES ALIMENTARIAS LATINOAMERICANAS POR MOTIVOS RELACIONADOS CON MEDIDAS TÉCNICAS NO ARANCELARIAS.

Sofía Boza, Juan Rozas e Rodolfo Rivers

América do Sul em face dos tratados bilaterais de inVESTimento: RUMO aO RETORNo do EsTADO NA SOLUÇão DE CONTROvÉRSIAS? 133 Magdalena Bas

FutURo DE LOS SISTEMAS NACIONALES DE CIENCIA TECNOLOGÍA E INNOVACIÓN EN LA AGENDA ECONÓMICA DE AMÉRICA LATINA: DEFINIENDO CAMBIOS REGULATORIOS O PROTEGIENDO INVERSIONES 146

Rodrigo Corredor

EL PAPEL DE LAS INSTITUCIONES DE CONTROL FINANCIERO SOBRE LOS DERECHOS HUMANOS EN EL CONTEXTO LATINOAMERICANO 157 Jose Miguel Camacho Castro

CONVERGENCIA REgUlATORIA EN LA ALIANZA DEL PACÍFICO: UN CAPÍTULO INCONCLUSO 170 Rodrigo Polanco Lazo

O CONSTITUCIONALISMO E A COMUNITARIZAÇÃO NO DIREITO INTERNACIONAL: POSSIBILIDADES PARA O COMÉRCIO INTERNACIONAL?

Camilla Capucio

ESCASSEZ HÍDRICA E DIREITO INTERNACIONAL ECONÔMICO: O BRASIL COMO PROTAGONISTA NA TRANSFERÊNCIA DE ÁGUA PARA REGIÕES ÁRIDAS

Douglas de Castro

A Segurança energética como base para maior integração na América Do Sul: à espera DE UM TRATADO MULTILATERAL

Matheus Bassani

Outros Artigos. 246

As PRÁtICAS RESTRITIVAS DA CONCORRÊNCIA NO MERCADO DE CONTRATAÇÃo PÚBLICA EUROPEU .. 248 Alice Rocha da Silva e Ruth M. P. Santos 
Do TRANSNACIONAL PARA O NACIONAL: IOSCO, O MERCADO DE VALORES MOBILIÁRIOS BRASILEIRO E ACCOUNTABILITY

Salem Nasser, Nora Rachman e Viviane Muller Prado

MigRaÇÃo de TRABALHADORES INTELECTUAIS BRASILEIROS PARA O MERCADO INTERNACIONAL: IDENTIFICAÇÃO DE ATOS DE ALICIAMENTO DE EMPREGADOS E MECANISMOS LEGAIS PARA IMPEDIR A APROPRIAÇÃo TECNOLÓGICA E CONCORRÊNCIA DESLEAL ..........................................285 José Carlos Vaz e Dias e João Marcelo Sant'Anna da Costa

THE EASIER WAY TO HAVE "BETTER LAW"? THE MOST-SIGNIFICANT-RELATIONSHIP DOCTRINE AS THE FALLBACK CONFLICT-OF-LAW RULE IN THE PEOPLE'S REPUBLIC OF CHINA

Chi Chung

REFLEXOS JURÍDiCOS DA GOVERNANÇA GLOBAL SUBNACIONAL: A PARADIPLOMACIA E O DIREITO INTERNACIONAL: DESAFIO OU ACOMODAÇÃO 320

Valéria Cristina Farias e Fernando Rei

Matrizes Políticas da JUSTiÇA PENAL INTERNACIONAL. 341 Francisco Rezek

RESPONSABILIDAD INTERNACIONAL DEL ESTADO FRENTE A LUCHA CONTRA LA DISCRIMINACIÓN RACIAL Y ÉTNICA EN ESPAÑA. 348

Edilney Tomé da Mata e Eduardo Biacchi Gomes Correio

The Peace Process in Sierra Leone: an analysis on marriages between culture and CRIME

Gustavo Bussmann Ferreira

Funcionalização e expansão do Direito Penal: o Direito Penal negocial ...............376 Antonio Henrique Graciano Suxberger e Dermeval Farias Gomes Filho

Proteção Internacional do Consumidor e Cooperação Interjurisdicional .396 Héctor Valverde Santana e Sophia Martini Vial

The land rights of indigenous and traditional peoples in Brazil and Australia.... 418 Márcia Dieguez Leuzinger e Kylie Lyngard 
The reception of European ideas in Latin America: the issue of the German sources in Tobias Barreto, A Prominent nineteenth CENTURy BraZilian legal scholar........439 Arnaldo Sampaio de Moraes Godoy

Normas Editoriais ..................................................................................... 461 


\title{
Futuro de los sistemas nacionales de ciencia tecnología e innovación en la agenda económica de américa latina: definiendo cambios regulatorios o protegiendo inversiones*
}

\author{
Future of national systems of science \\ technology and innovation in the ongoing \\ economic agenda: leveraging regulatory shift \\ or protecting investments
}

Rodrigo Corredor**

\section{Resumen}

A lo largo de la última década los caminos para lograr el crecimiento económico en Latinoamérica han mostrado una convergencia hacia la inversión en CT\&I lo cual se ha visto reflejado en el fortalecimiento de las estructuras administrativas de las instituciones nacionales encargadas de tales temas. Sin embargo, lograr los cambios estructurales que alienten la competitividad y la participación del sector empresarial local en el escenario de la economía del conocimiento y preponderancia de cadenas globales de valor, parece seguir siendo un desafío que los países de la región han asumido con mas voluntad y medios que visión y creatividad. En efecto, los enfoques y estructuras casi idénticas que exhiben varios países de la región en esta materia, denotan que la finalidad por impulsar políticas públicas en materia de CT\&I ha sido interpretada más como el mandato de emular experiencias exitosas de países industrializados, sin tomar en consideración los rasgos específicos que deben caracterizan estos programas. Desde esta perspectiva la reflexión se plantea en torno a la idea de seguir la línea de crecimiento que ha conducido a las economías actuales por la ruta del crecimiento en detrimento de factores sociales y ambientales. ¿es esto una fatalidad para los países emergentes?; o por el contrario, ¿tienen éstos la obligación de plantear una nueva lógica que privilegie el aprovechamiento sostenible de sus abundantes recursos así como la fragilidad de los ecosistemas donde éstos se encuentran ubicados?. Ambos interrogantes conducen a una respuesta: la región está llamada a liderar una interpretación renovada de las normas del sistema multilateral del comercio, y un punto de partida puede ser la relectura de los planteamientos sobre los alcances de la actividad inventiva y sus vínculos con el sector industrial y estatal, de manera que los recursos públicos destinados a I+D se enfoquen hacia la solución, control o mitigación de los efectos devastadores heredados de la etapa de capitalismo avanzado.

Palabras clave: Políticas de ciencia y tecnología. Crecimiento económico. Incentivos. Inversión extranjera directa.

** Abogado de la Universidad Externado de Colombia, Especialista en Propiedad Intelectual de la misma universidad y Especialista en Gestión Pública e Instituciones Administrativas de la Universidad de los Andes. Master en Derecho Económico Internacional de la Universidad de Berna (Suiza). Investigador del Departamento de Derecho Económico de la Universidad Externado de Colombia. Correspondencia: Departamento de Derecho Económico Universidad Externado de Colombia, Carrera 3 Este No. 9-96, primer piso, Bogotá, Colombia. E-mail: rodrigo.corredor@ uexternado.edu.co 


\section{Abstract}

Over the last decade the paths to achieve economic growth in Latin America have shown a convergence in upgrading science, technology and innovation (ST\&I). This fact is reflected in the strengthening of the administrative structures of national systems along the region. However, the almost identical structures of these programs in the region show that the purpose of promoting S\&T as a public policy has been interpreted often, as a mandate to emulate successful experiences of industrialized countries without taking into account the country-specific nature that should characterize these programs. Is this a fatality for developing countries? Or conversely, do they have an obligation to foster a new logic that favours the sustainable use of its abundant resources of the ecosystems where they are located? Both questions leads to one single answer: the region is called to lead a renewed interpretation of the rules of the multilateral trading system, namely those related to the creative industries and its links with industrial sectors, in a way such that resources for $\mathrm{R}+\mathrm{D}$ can also be directed towards the solution, control or mitigation of the devastating effects inherited from the advanced capitalism period.

KeyWords: Science and technology policy. Economic growth. Incentives. Foreign direct investment.

\section{INTRODUCCIÓN}

A lo largo de la última década los caminos para lograr el crecimiento económico en Latinoamérica muestran una convergencia hacia la inversión en Ciencia Tecnología e Innovación (CT\&I) lo cual se ha visto reflejado en el fortalecimiento de las estructuras administrativas de las instituciones nacionales encargadas de tales temas. Sin embargo, lograr los cambios estructurales que alienten la competitividad y la participación del sector empresarial local en el escenario de la economía del conocimiento y preponderancia de Cadenas Globales de Valor (CGV), parece seguir siendo un desafío que los países de la región han asumido con mas voluntad y medios que visión y creatividad.

En efecto, los enfoques y estructuras casi idénticas que exhiben varios países de la región en esta materia, denotan que la finalidad por impulsar políticas públicas en materia de CT\&I ha sido interpretada más como el mandato de emular experiencias exitosas de países industrializados, sin tomar en consideración los rasgos específicos que deberían caracterizar estos programas en la región.

Esta fenomenología de los Sistemas Nacionales de Ciencia Tecnología e Innovación (SNCT\&I) en Latinoamérica adquiere matices interesantes, en momentos en los que el crecimiento de la región parece detenerse ante la desaceleración de la economía mundial y éstos países tendrán que concentrar sus esfuerzos en mitigar los efectos de la inevitable contracción de los ingresos derivados de las actividades extractivas con base en las cuales han planteado la financiación de tales sistemas. El panorama descrito nos sugiere que los problemas a solventar estarán relacionados con la sostenibilidad financiera, académica y/o científica de los programas de investigación, con lo cual se corre el riesgo no sólo de continuar estancados en los rankings globales de innovación, sino además de haber

Puntuales - la promoción de clústeres de innovación y las deducciones tributarias a actividades de CT\&I. En la tercera parte, se aborda la potencialidad o eventualidad de que los temas relacionados con la promoción a la CT\&I caigan dentro de la esfera de lo que se denomina el "interés público" en el marco de controversias Estado-Inversionista. Por último, se proponen soluciones de cara al panorama de contracción de las rentas provenientes de industrias extractivas haciendo énfasis en la necesidad de que las políticas de innovación recuperen una interpretación compatible con el desarrollo económico que requieren los países en vías de desarrollo y de la cual existe el riesgo de alejarse en el panorama actual de prevalencia de acuerdos de promoción y protección de inversiones que desdibujan los consensos sobre los cuales se ha edificado el sistema multilateral de comercio.

\section{NarRativa de la fORMULACIÓN DE POLÍTICAS PÚBLICAS EN MATERIA DE INNOVACIÓN}

Aunque existen mediciones periódicas de los indicadores de CTI\&I en la región, ${ }^{1}$ muy poco se habla de

1 RED DE INDICADORES DE CIENCIA Y TECNOLOGÍA IBEROAMERICANA E INTERAMERICANA. Red de indicadores de ciencia y tecnología iberoamericana e interamericana. 2014. Available in: 
la composición de las rentas que sustentan estas actividades. Sin embargo, la reconocida importancia que las economías de la región han dado en los últimos años a sus sectores extractivos (hidrocarburos, minerales y en algunos casos agricultura intensiva) y la situación de fuerte dependencia de las rentas generadas por éstos sectores, ha generado serios procesos de reflexión al interior de los Gobiernos nacionales sobre la necesidad del fortalecimiento de la CT\&I como motor del desarrollo económico y en esa medida influenciaron el aumento de las partidas presupuestarias destinadas a actividades relacionadas con la CT\&I. ${ }^{2}$

En efecto, durante la reciente bonanza económica experimentada en la región, ${ }^{3}$ el aumento de las ganancias en el sector extractivo se vio reflejada en una tendencia generalizada hacia la búsqueda de participaciones más importantes de las rentas generadas por la explotación de sus recursos naturales con el fin de soportar sobre dicha base nuevos sectores de la economía. ${ }^{4}$ La creación

<http://www.ricyt.org >. Adelanta mediciones con base en Indicadores de Ciencia y Tecnología definidas para Ibero y Latinoamérica. 2 En Colombia la Ley 1286 de 2009 y de manera especifica el Acto Legislativo 5 de 2011 (julio 18) artículo 2. señala que los recursos de inversión del Sistema General de Regalías se destinarán al financiamiento de proyectos para el desarrollo social, económico y ambiental de las entidades territoriales; al desarrollo de inversiones físicas en educación e inversiones en materia de ciencia, tecnología e innovación y, en general, al aumento de la competitividad general de la economía buscando mejorar las condiciones sociales de la población. Una redaccion similar tiene la Ley peruana 28303 de 2004, la cual en su artículo 26 dispone : "El Plan Nacional de CTel es el instrumento de propuesta y ejecución de la política nacional de CTel, forma parte de las políticas de Estado y responde a una visión geoestratégica del corto, mediano y largo plazo. Tiene como base para su elaboración el establecimiento de líneas estratégicas, la fijación de prioridades y el diseño y la articulación e implementación de programas nacionales, regionales y especiales propuestos por los sectores y entidades que conforman el SINACYT". En Mexico, De acuerdo al artículo 11 de la Ley Orgánica del Consejo Nacional de Ciencia y Tecnología El patrimonio del CONACyT se integrará con: I. Los bienes muebles e inmuebles que le asigne el Ejecutivo Federal, y los que adquiera por cualquier título legal, y II. Con las transferencias, los subsidios, participaciones, donaciones y legados que reciba y, en general, con los ingresos que obtenga, por consultas, peritajes, regalías, recuperaciones, derechos propiedad intelectual o cualquier otro servicio o concepto propio de su objeto.

3 La evolución positiva del PBI propició un aumento de los recursos económicos destinados a I+D. La inversión en I+D para ALC en 2012 superó los 54 mil millones de dólares (medidos en PPC), duplicando los valores con los que inició la serie en 2003. http:// www.ricyt.org/files/Estado $\% 20 \mathrm{de}^{2} \% 201 \mathrm{a} \% 20$ Ciencia $\% 202014 /$ Estado_de_la_ciencia_en_imagenes(1).pdf

4 En Colombia, la propia redaccion del Artículo 361 Constitucional - que sirve de fundamento a la financiacion de las actividades de CT\&I - refleja la fuerte dependencia hacia las actividades del sector extractivo cuando señala: "Con los ingresos provenientes de las de herramientas fiscales y el recurso a entornos contractuales vinculados a la transferencia de tecnología, como incentivos para la inversión, también hace parte del conjunto de herramientas que, por la vía de las recomendaciones de organismos multilaterales y regionales, se han venido replicando en varios países de américa latina, casi siempre enmarcadas o presentadas dentro de esquemas de fortalecimiento a los SNCT\&I. ${ }^{5}$

Desde el punto de vista de la arquitectura institucional, estos sistemas han sido concebidos dentro de la idea de provocar una inserción en la economía del conocimiento $^{6}$ y lograr un salto cualitativo que permita emular el éxito de las económicas asiáticas en la década de los noventa, ${ }^{7}$ con la diferencia sustancial de que

regalías que no sean asignados a los departamentos y municipios, se creará un Fondo Nacional de Regalías, cuyos recursos se destinarán a las entidades territoriales en los términos que señale la ley. Estos fondos se aplicarán a la promoción de la minería, a la preservación del ambiente y a financiar proyectos regionales de inversión definidos como prioritarios en los planes de desarrollo de las respectivas entidades territoriales". Subrayado fuera de texto.

5 Un reciente documento del Banco Mundial pone de presente esta tendencia al afirmar: "[...] las prioridades de las políticas económicas en la región han evolucionado respondiendo a los cambios globales a medida que estos cambios han exacerbado ciertas dificultades de larga data en la región, como su dependencia de las materias primas minerales y agrícolas y sus tasas de ahorro comparativamente bajas. Por lo tanto, es probable que en la región se intensifique el debate sobre las prioridades de las políticas públicas en el contexto de un nuevo panorama global y que tenga como punto central la agenda del crecimiento."

6 Por ejemplo: Chile cuenta hoy con una Estrategia Nacional de Innovación para la Competitividad que propone "[...] avanzar decididamente al desarrollo por la senda de la Economía del Conocimiento, combinando para ello las ventajas del modelo exportador de recursos naturales, donde el país tiene ventajas importantes y ha logrado ya grandes avances, con las habilidades creadas por un esfuerzo creciente en la generación de capital humano y conocimiento que, aplicados al proceso productivo, permitan desarrollar sectores mas sofisticados y de alta competitividad a nivel global", pagina 29, documento del consejo nacional de innovación para la competitividad cna.gov.co. de acuerdo con BANCO DE DESENVOLVIMENTO DA AMÉRICA LATINA. Regulación de la inversión extranjera en América Latina. Regulaciones y opciones para reformas al clima de inversión. 2014. (Serie Políticas Públicas y Transformación Productiva $\mathrm{N}^{\circ} 14$ ) olombia, por su parte " ha concedido exenciones al impuesto a la renta de 10, 15 y 30 años, respectivamente, para las inversiones en silvicultura, generación de energía eléctrica (biomasa, solar, eólica) y para la construcción. Asimismo, el Gobierno colombiano prepara una ley para autorizar la inversión privada y la extranjera en instituciones públicas y privadas de educación superior." p. 30.

7 De acuerdo con CEPAL Mientras la contribución de estas economías al crecimiento del PIB mundial se duplicó con creces entre los períodos 1990-1995 y 2005-2012, aumentando del 19\% al $41 \%$, la contribución de América Latina se mantuvo estable, pasando del $8 \%$ al $9 \%$. El notable desempeño de Asia se relaciona 
muchos factores, que hoy en día distorsionan el cumplimiento de tales fines, no existían o simplemente comenzaban a esbozarse en el panorama económico regional asiático de aquella época, ${ }^{8}$ por lo cual no fueron un obstáculo impediente, como si parecen serlo hoy la excesiva oferta de productos tecnológicos situados muy lejos de la frontera tecnológica en la cual los países logran niveles aceptables de absorción de la tecnología a la que tienen acceso o la preponderancia del sector servicios en la economía global. ${ }^{9}$

En este sentido, el tímido retorno a una narrativa de corte intervencionista - implícita en el discurso que hoy acompaña la promoción de SNCT\&I en la región - puede de cierta manera ser interpretado como una forma propiciar la conformación de sectores producción de medios de producción para resolver lo que Fajnzylber calificó en su momento como «industrialización trunca» y que a su vez sirvió de base a la útil distinción entre «proteccionismo para el aprendizaje», que caracterizó a los procesos industrializadores más exitosos del Asia Oriental, y el «proteccionismo frívolo», que en América Latina signó a la industrialización tardía. ${ }^{10}$

directamente con la irrupción de China, país que se ha convertido en la segunda economía mundial y el principal exportador mundial de bienes y está próximo a ser una potencia tecnológica. Su tasa de crecimiento, del 10\% anual durante más de tres décadas, no tiene parangón histórico y menos aún en el caso de una nación tan poblada." 8 En este sentido Arocena, R; Sutz, destacan como "El modelo de industrialización fue muy distinto en Asia Oriental y América Latina. Si bien ambos tuvieron en común la importancia del rol desempeñado por el Estado, las diferencias en el desempeño de ese rol fueron realmente muy grandes. Así por ejemplo no se conoció en nuestro continente nada parecido al relacionamiento entre Estado y tecnología en Japón. Allí, antes de la liberalización de fines de los '60, no se decidía ninguna compra de tecnología, "joint venture" o acuerdo de patentes sin aprobación del MITI, que procuraba activamente mejorar los términos e cada convenio, y no permitía incorporar tecnología extranjera si no estimaba que la industria nacional involucrada estaba madura para aprovecharla"

9 A diferencia de Asia, donde el desarrollo tecnológico estuvo ligado al fortalecimiento del mercado interno, en momentos en los que la tanto la oferta de productos tecnológicos - poco avanzados - era relativamente baja a nivel mundial y no consideraba a tales países como mercados objetivo; América Latina, afronta no sólo la sobreoferta de productos tecnológicos sino que por efecto de la liberalización de los servicios, buena parte del capital humano que podría dedicar su esfuerzo y capacidades a labores científicas, encuentra más atractivo asumir posiciones de tipo gerencial dentro de empresas multinacionales. Esta situación hace aún más difícil que las políticas públicas de ST\&I encuentren el espacio para lograr adaptar el proceso endógeno de innovación a la demanda interna.

10 AROCENA, R; SUTZ, J. Desigualdad: tecnología e innovación en el desarrollo latinoamericano Iberoamericana. Nueva época, 2001. p. 36
Desde esta perspectiva, es posible afirmar que el objetivo principal de esta nueva generación de políticas de ST\&I es lograr profundizar la capacidad endógena para la innovación, lo cual aparece como una tarea pendiente en el contexto latinoamericano. Sin embargo es necesario precisar que en el contexto actual, la señalada dependencia de la rentabilidad del sector extractivo en temas asociados con la financiación de los SNCT\&I, plantea nuevos problemas como los señalados por Mazzucato quien advierte como este tipo de actividades de innovación - se caracterizan por ser de alto riesgo y tener tasas de retorno de mediano o largo plazo, por lo cual una ruptura en los flujos de capital que sostienen la investigación pueden resultar poco favorables para la sostenibilidad de los proyectos y por ende de fragilizar la gestión de todo el sistema en su conjunto. ${ }^{11}$

\subsection{Cambios y continuidad entorno al concepto de innovación}

Pero más allá de las probables fallas que puedan presentarse en el diseño de políticas públicas en materia de CT\&I, el contexto actual exige abordar la innovación como concepto eminentemente económico, superando las visiones en las que se encuentran inmersas las instituciones políticas y sociales que directamente inciden en la preservación de un imaginario colectivo que subvalora a la tecnología y por ende dificulta el progreso tecnológico. ${ }^{12}$

Igualmente resulta fundamental reconocer como el cambio en las relaciones industriales introducido entre

11 MAZZUCATO, M. The entrepreneurial state: debunking public vs. private sector myths. Londres: Anthem, 2013. p. 182

12 En el mismo sentido AROCENA, R; SUTZ, J. Desigualdad: tecnología e innovación en el desarrollo latinoamericano Iberoamericana. Nueva época, 2001. complementan: "Esto último hunde sus raíces en el pasado lejano, pues constituye una herencia de la colonización ibérica, que impuso pautas culturales muy distintas a las trasplantadas a las zonas de colonización anglosajona. La España que conquistó América fue la que se arruinó a sí misma, cultural y técnicamente, destruyendo la agricultura morisca, expulsando a los judíos, sometiendo la sociedad a la Inquisición. Su impronta entre nosotros se manifestó en las universidades monásticas que perduraron bastante más allá de la independencia, en el desprecio al trabajo manual, en el parentesco entre las escuelas de oficios y los establecimientos correccionales. La cultura oficial asignó un lugar marginal y - salvo en el caso de la biomedicina y la investigación agropecuaria más bien decorativo a la ciencia, pese a esfuerzos y logros relevantes, y prácticamente dejó afuera a la tecnología. Causa y consecuencia de tal fenómeno,el "matrimonio entre la ciencia y las artes útiles", que caracteriza a la Segunda Revolución Industrial, en estas tierras apenas si se consumó." 
otras por la incursión de las nuevas tecnologías, el desarrollo de los medios de comunicación y la desmaterialización progresiva de los intercambios comerciales viene propiciado cambios profundos en lo que entendemos por innovación y los mecanismos legales para estimularla. ${ }^{13}$ Un ejemplo de ello es la concesión de derechos exclusivos que como en el caso de las patentes, en los albores de la revolución industrial, fueron empleadas como medio para proteger a los inventores del riesgo de que sus creaciones divulgadas en el marco de las exposiciones universales fueran objeto de copia por otros empresarios.

En efecto, aunque en la actualidad, la existencia del riesgo a "ser copiado" persiste como un rezago de la interpretación rigurosa de las instituciones de protección de DPI's, las discusiones sobre el enfoque económico de las políticas de innovación permiten apreciar un giro hacia la necesidad de facilitar la emulación y de integrar los aparatos productivos de los países en desarrollo a la actividad inventiva de las Empresas Multi Nacionales (EMNs) lideres en innovación. De esta manera, podemos hablar de un cambio en el enfoque respecto a la actividad creativa que sobrepasa la necesidad de la protección de la invención y que parece enfocarse más hacia el estímulo continuo de sinergias benéficas (entornos colaborativos) los cuales son percibidos como escenarios ideales para el desarrollo sostenible de la actividad innovadora. ${ }^{14} \mathrm{El}$ marco en el que se plantea esta nueva

13 Esta realidad se refleja en la discusion actual de la Politica Nacional de Ciencia, Tecnologia e Innovacion 2015-2025 en la cual se deja en claro que "El diseño de la política de CTI a partir del concepto de SNI va más allá del enfoque lineal tradicionalmente utilizado en los países de América Latina, en el que la innovación empresarial se considera como la última etapa de un proceso ordenado, que inicia con la investigación básica, pasa a la investigación aplicada y luego al desarrollo tecnológico que es incorporado en la producción (BM 2008; BID 2014). La principal influencia del concepto de SNI en el diseño de la política pública consiste en la adopción de un enfoque más amplio que el tradicional de fallas de mercado, LIZU$\mathrm{KA}$; Soete. Catching up in the twenty first century: globalization, knowledege and capacities in latin america: a case for natural resource based activities. [Amsterdã]: Unu_Merit Working Paper Series, 2011. Este enfoque determina que no son solo las fallas de mercado las que ameritan intervenciones gubernamentales, sino que la naturaleza de interacción de la CTI y la variedad de sus instituciones hacen que las fallas de Estado y las fallas sistémicas sean otros factores determinantes del diseño de la política."

14 Para autores como MOWERY, D.; SIMCOE, T. The Internet. In: STEIL, B.; VICTOR, David G.; NELSON, R. Technological innovation and economic performance. Princeton: Princeton University Press, 2002. "national IPR systems and national economies and innovation systems "co-evolve" in a complex, path dependent, and iteractive fashion." relación obviamente está influenciado por el desarrollo del derecho económico internacional y de manera mas reciente por la preponderancia que ha logrado la Inversión Extranjera Directa (IED) como medio de movilización y optimización de los factores de producción.

\subsection{La innovación: un concepto para armar}

Dentro de las criticas más recurrentes a las economías de la región está la de su incapacidad para generar innovación y de vincularla al desempeño sostenible de la economía, es decir, de integrar la agenda de investigación y desarrollo con los esfuerzos desplegados en el plano industrial a través de otras políticas públicas tales como educación, la salud, la seguridad, la protección del medio ambiente o el desarrollo de proyectos de infraestructura etc. De acuerdo con Gervais, podría afirmarse que esta situación es, de cierto modo, el resultado de la aplicación de teorías que llevaron a estos países a implementar trasplantes normativos en materia de protección de DPIs bajo supuestos que hoy se presentan como erróneos o por lo menos imprecisos. El mencionado autor señala:

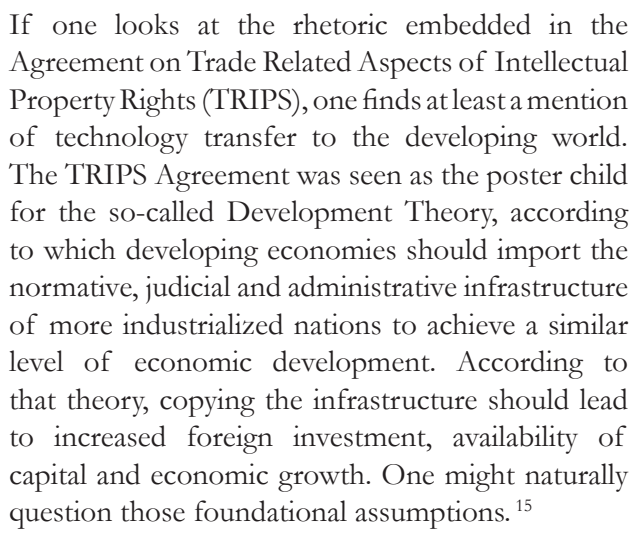

Ahora bien, algo similar parece estar sucediendo con el concepto de innovación, el cual ha terminado por ser incluido como elemento central en las estrategias de transformación productiva de buena parte de los países latinoamericanos. Dentro de esta comprensión, dichas políticas incluyen como elemento catalizador a la promoción de la Inversión Extranjera (IED), atribuyéndole la capacidad de lograr niveles mejorados de interacción entre las firmas extranjeras y los actores internos, los

15 GERVAIS, Daniel. Current issues in international intellectual property norm-making. In: DREXL, J. et al. (Ed.) Eu bilateral trade agreements and intellectual property: 3 for better or worse? MPI Studies on Intellectual Property and Competition Law 20. Berlin: SpringerVerlag, 2014. p. 5. 
cuales, estimulados por la deslocalización de la producción, deberían normalmente terminar por participar armónicamente dentro del ciclo productivo. ${ }^{16}$

Pero la realidad es diferente. En efecto, la mayor parte de lo que los países latinoamericanos contabilizaron durante el reciente periodo de bonanza económica como innovación, corresponde verdaderamente al despliegue de un tipo de IED enfocada exclusivamente a la deslocalización de actividades relacionadas con la manufactura o ensamble de ciertos productos tecnológicos hacia países con menores costos de producción. ${ }^{17}$ Para revertir esta tendencia, en la actualidad muchos países parecen estar apartándose de este tipo actividades que prevén niveles controlados de transferencia de tecnología, vía gestión de DPI's - y confiriendo una mayor importancia al valor agregado que los sectores manufactureros pueden aportar a los productos de sus canastas exportadoras a través de la incorporación de la CT\&I para de esta manera comenzar a reducir las brechas tecnológicas.

Desde el punto de vista del diseño de políticas de desarrollo productivo, es claro que las decisiones respecto de la naturaleza de las inversiones que se requieren para insertarse en la economía del conocimiento no pueden quedar exclusivamente en manos de los inversionistas privados/extranjeros, puesto que estos se enfocaran en innovaciones que busquen mejorar su propia eficiencia, esto es, en la optimización de los factores de producción bajo la premisa esencial de "producir más con menos".

Es aquí donde radica el contrasentido del contexto en el que se dan actualmente las políticas de innovación en Latinoamérica, pues por un lado, éstas se enfocan en

16 Esta Vision se refleja en varios documentos orientadores publicados por organismos multilaterales, tales el Banco Mundial, en el cual se afirma:"El aumento de los flujos financieros entre los países, sobre todo de la IED, podría ser impulsado por empresas que buscan capitalizar a partir de las mejoras de eficiencia facilitadas por la fragmentación de las etapas de producción en diferentes países. Por lo tanto, la creciente participación del Sur en los flujos financieros globales podría ser un motor potencial de crecimiento económico. Dichos flujos pueden no solo aliviar las restricciones financieras en las economías receptoras sino también ser un vehículo de difusión tecnológica y de conocimientos. De hecho, los responsables de las políticas del Sur, incluyendo a ALC, ven el atractivo de la IED y las corporaciones multinacionales como una prioridad de las políticas." 17 Por su parte UNCTAD pone en claro como en las etapas tempranas del desarrollo tecnológico, las empresas no requieren como tal la implementación de departamentos de I\&D, y por lo tanto la IED de la que son receptoras se enfoca hacia actividades de bajo valor agregado que solo inciden en la reducción de los costos de la empresa que deslocaliza su producción. promover el acceso ampliado a los recursos naturales al tiempo que atribuyen a dichas inversiones un efecto positivo en materia de innovación. En este sentido, resulta pertinente el argumento avanzado por Mezue y otros en el sentido de que:

\begin{abstract}
"Economists have long wondered why nations endowed with oil (such as Iran, Iraq, Mexico, Nigeria, and Venezuela) or precious metals (such as Mongolia, Peru, and Russia) generate billions upon billions in revenues and profits yet manage to create few jobs and little national economic growth. The answer is that investments in resource industries in developing nations lead to efficiency innovations, designed to produce more with less. From the day these rigs and refineries go into operation, the objective of their managers is to increase productivity by reducing employment. This is the logic of efficiency innovation, and its outcome is net job loss, not gain". ${ }^{18}$
\end{abstract}

Es claro que esta visión así como la tendencia a privilegiar el uso de rentas públicas para el financiamiento de la SNCT\&I está ligada a una comprensión coyuntural de concepto de innovación, la cual se refleja en el uso efectista que se hace del mismo dentro del discurso político actual. Esta situación pone de presente problemas más complejos relacionados con la vinculación tardía del concepto de innovación a políticas de desarrollo productivo, así como con las dificultades para integrar los efectos sociales de otros temas transversales tales como la gestión de los Derechos de Propiedad Industrial (DPI's) o el derecho de la competencia, lo que refleja en últimas un aislamiento de la actividad innovadora de las discusiones que son pertinentes para los sectores manufactureros.

\section{Panorama de los estímulos a la INNOVACIÓN}

En Latinoamérica, la manera como se formula la innovación como objetivo dentro de políticas públicas está influenciada principalmente por la comprensión de los efectos sociales que se reconocen a ciertas categorías de DPI's. De allí, la insistencia en el fomento de los vínculos entre la academia y la empresa así como de la intención de sopesar la efectividad de tales sinergias

18 MEZUE et al. The power of market creation: how innovation can spur development, foreign affairs, Enero de 2015. Available in: $<$ m.foreignaffairs.com/articles/142495/bryan-c-mezue-clayton-mchristensen-and-derek-van-bever/the-power-of-market-creation>. 
usando indicadores (como el número de patentes solicitadas/concedidas) y de atribuir a tales mediciones la potencialidad de facilitar las decisiones a los inversionistas foráneos, que como se ha dicho, son percibidos como factores determinantes del proceso innovador.

Dos herramientas son claramente distinguibles: los denominados clústeres innovadores y los estímulos tributarios a las actividades de CT\&I. Los dos hacen parte de estrategias que toman como punto de partida la existencia de sectores dinámicos que pueden mejorar su desempeño mediante el apalancamiento de actividades $I \& D$, haciendo posible inducir niveles adicionales de transformación productiva.

En el caso de los clústeres, las empresas que los conforman se benefician al poder actuar más productivamente en el abastecimiento de insumos, y lograr un acceso directo a la información y a la tecnología. En este sentido es posible distinguir tres elementos de éxito de cualquier política clúster: la asociatividad y presencia de redes, la tecnología de innovación y el capital humano, las cuales deben conjugarse de manera armoniosa con el fin de elevar la productividad del sector seleccionado. ${ }^{19} \mathrm{El}$ resultado esperado de este tipo de políticas es la consolidación de núcleos productivos altamente institucionalizados y con capacidad de coordinar el desempeño industrial con miras a posicionar productos y/o servicios tanto en el mercado interno como a nivel internacional.

Por su parte Rodrick, D. identifica tres áreas para la consolidación de una política eficiente de clústeres, la primera esta relacionada con la mejora de la productividad de los factores: capital humano y físico. ${ }^{20} \mathrm{La}$ segunda, más del resorte de las instituciones públicas encargadas, que consiste en procurar una mejora del entorno de negocios a través de unos indicadores macroeconómicos estables y la implementación de regulación pertinente. Por último, es necesario que quienes están asociados procuren resolver las fallas del mercado que se vayan detectando para de esta manera conservar las condiciones que hacen atractivo el clúster a empresas del sector privado o que tienen la potencialidad de atraer nuevas sinergias y oportunidades de transferencia de tecnología.

19 FEDESARROLLO. 20 años de politicas de competitividad en Colombia. Bogotá. 2013.

20 RODRIK, D. One economics: many récipes. Princeton: Princeton University Press, N. J, 2007.
En cuanto a las deducciones tributarias por inversión en CT\&I, para el caso colombiano, el documento Conpes 3834 las define como aquellos gastos que se descuentan de los ingresos y que afectan la utilidad, generando un menor impuesto a pagar por parte del contribuyente. De esta forma, al fomentar las actividades de $\mathrm{I}+\mathrm{D}+\mathrm{i}$, las deducciones se convierten en un instrumento de financiamiento público indirecto que apoya la competitividad empresarial. Un punto importante a resaltar en relación con este tipo de instrumentos es su disponibilidad tanto para empresarios locales como a empresas extranjeras que deseen aprovechar oportunidades de inversión o que busquen provocar la integración de productores locales en su cadena de valor.

La necesidad de este tipo de instrumentos suele estar justificada en fallas de mercado que a su vez están influenciadas, como ya se mencionó, por la errónea concepción del efecto social de las patentes como medio de protección de las actividad inventiva desplegada por actores privados. En efecto, el carácter de bien público no excluible que se reconoce al conocimiento, dificulta la apropiación absoluta de los beneficios que se generan de una invención y por consiguiente sirve de causa eficiente a una segunda falla de mercado que consiste en la incertidumbre que se genera en torno a la protección y al riesgo inherente de la actividad innovadora.

Aunque las deducciones tributarias parecen acertar como mecanismo para mitigar los costos sociales que surgen de la exacerbada tendencia a la privatización del conocimiento que ha caracterizado el reciente periodo de la globalización, a su implementación se le reconocen un alcances limitados. El documento Conpes citado reconoce cuatro áreas problemáticas:

\begin{abstract}
“i) los niveles de inversión privada en el financiamiento de I+D son bajos; ii) los porcentajes de deducción efectivos pueden llegar a ser insuficientes para compensar los riesgos asociados a las inversiones en $\mathrm{I}+\mathrm{D}+\mathrm{i}$; iii) los usuarios del instrumento se concentran en grandes empresas y en pocos sectores, dejando por fuera a las pequeñas y medianas empresas que no declaran renta líquida, así como a sectores productivos que realizan $\mathrm{I}+\mathrm{D}+\mathrm{i}$ en menor escala; y iv) el cupo global de deducción ha sido subutilizado."21
\end{abstract}

Esta evaluación pone de presente un aspecto relevante, cual es el hecho de que hasta el momento la

21 Departamento Nacional de Planeación. Documento Conpes 3834, Lineamientos de Política para Estimular la Inversión Privada en Ciencia, Tecnología e Innovación e Través de Deducciones Tributarias, Bogotá: 2015, p. 15. 
utilización del instrumento ha estado concentrado en las "grandes empresas", dentro de las cuales debemos incluir las actividades EMNs que se benefician de las deducciones tributarias registrando como proyectos de $\mathrm{I}+\mathrm{D}+\mathrm{i}$, sus actividades de transferencia de tecnología. Por esta razón, es importante afinar los mecanismos de medicion tanto de la cantidad como de la calidad de innovacion que se puede dar en contexto de la IED, ${ }^{22} \mathrm{Au}-$ tores como Lizuka y Soete afriman que las patentes solo muestran el nivel de conocimiento codificado lo cual es insuficiente para establecer la ocurrencia de interacciones positivas que estimulen el flujo de conocimiento relevante para el desarrollo de ciertos sectores. la creacion de conocimiento requiere ciertamente de Sistemas Nacionales de CT\&I pero dentro del concepto que ellos denominan «Knowledge Creation Systems». ${ }^{23}$

El analisis precedente, pone en evidencia cómo la presión por incorporar la noción de innovación dentro de las políticas públicas esta conduciendo a las administraciones nacionales a escenarios mas propios innovación basada en la eficiencia, con el agravante de que los estímulos públicos a la I+D se han ido a fortalecer estructuras de mercado para agentes que no las requieren.

\section{La INNOVACIÓN COMO PARTE deL 'INTERÉS PUBLICO' EN EL MARCO DE CONTROVERSIAS EstADO-INVERSIONISTA}

El panorama esbozado hasta el momento, plantea una situación en la cual el Estado debe asumir un rol activo en materia de políticas de CT\&I, asegurando que el marco regulatorio y las medidas de estímulo a la innovación no resulten discriminatorias frente a la actividad de los inversionistas extranjeros. ${ }^{24}$ En este contexto, la

22 Estimular la protección y observancia de los DPI's no equivale necesariamente a estimular los SNCT\&I. Las cifras que reflejan aumentos en el número de de patenes solicitadas y/o concedidas no implican necesariamente que se estén generando ciclos virtuosos entre el sector manufacturero local y los inversionistas foraneos, más aún cuando la mayoria de tales inversiones extranjeras consisten en actividades de tipo extractivo con bajos niveles de transferencia de tecnologia.

23 LIZUKA; Soete. Catching up in the twenty first century: globalization, knowledege and capacities in latin america: a case for natural resource based activities. [Amsterdã]: Unu_Merit Working Paper Series, 2011.

24 De acuerdo con el Banco Mundial "Los países en ALC ya han adoptado algunas políticas industriales, como la promoción de la inversión y el comercio que tiene como objetivo ciertos tipos de empresas e industrias y no otras. Una ampliación de este debate podría definición de marcos regulatorios que apoyen, dentro del mercado interno, la integración progresiva a CGV, o la configuración de entornos colaborativos intensivos en transferencia de tecnología, podrían beneficiarse de la implementación de esquemas menos ortodoxos en cuanto a la gestión de DPI's, pero que a su vez resulten compatibles con la tendencia global a impulsar estándares de protección y observancia mas rigurosos como los que actualmente parecen estar negociándose en los llamados acuerdos megaregionales. ${ }^{25}$

Dentro de las soluciones que acompañan el desarrollo de estrategias de mercado basadas en la innovación está el recurso al subsidio de actividades exportadoras o a la I+D. En el primer caso, la reglas del comercio internacional restringen fuertemente el uso de subsidios a las exportaciones razón por la cual las políticas publicas parecen dar prevalencia al apoyo a la $\mathrm{I}+\mathrm{D}$, bien sea integrando la CT\&I a sectores con cierto grado de desarrollo, como en el caso de los clústeres; o bien, con énfasis en la promoción de la innovación enfocada hacia la solución, de los problemas básicos que aquejan a millones de habitantes en los países en desarrollo. Esta última perspectiva resulta interesante en la medida en que reconoce la existencia de un mercado que demanda activamente soluciones técnicas, el cual puede ser servido por la vía de estímulos públicos a emprendedores locales, todo dentro de una visión de la innovación más adaptada al contexto social y económico que exhibe la región después de la bonanza de los comodities.

Sin embargo, las estrategias de este tipo requieren de un cierto margen de planificación estatal que permita no sólo programar los incentivos sino, estructurar el mercado con miras a la satisfacción de las necesidades relevantes en diversas áreas prioritarias. Es aquí donde surge la potencialidad de que los temas relacionados con la CT\&I caigan dentro del ámbito de los temas de interés público que pueden verse afectados en el marco de controversias Estado-Inversionista. ${ }^{26}$

abarcar los incentivos basados en políticas, entre ellos las políticas tributarias y de gasto, teniendo presente la ayuda a los mercados para internalizar las grandes externalidades positivas asociadas con la investigación y el desarrollo $(\mathrm{I}+\mathrm{D})$ y la adopción y adaptación a nuevas tecnologías."

25 Haciendo referencia a los dos grandes acuerdos actualmente en negociación Trans-Pacific Partnership (TPP) y Transatlantic Trade and Investment Partnership (TTIP) Calverley señala: "While tariff reduction on goods was the main focus of trade deals in the 20th century, the new generation of pacts address "behind the border" obstacles such as restrictions or biased procurement policies, arcane investment rules and weak intellectual property protection."

26 Aunque puede aparecer a primera vista como una hipótesis 
Como es bien sabido las grandes corporaciones multinacionales persisten en su empeño por explotar todas las definiciones posibles de expropiación como una manera de exigir compensaciones ante los eventuales cambios regulatorios propuestos/planeados por los Gobiernos. En el caso de las políticas públicas de CT\&I existe un riesgo de que al vincular las actividades de $\mathrm{I}+\mathrm{D}$ a la protección de la salud, la biodiversidad o la generación de energías renovables, mediante el uso de subsidios tanto al sector público como a las empresas locales que buscan participar dentro el mercado a través de pequeñas innovaciones incrementales, se de pie a reclamaciones por parte de las grandes corporaciones que, en ciertos casos, cuentan inclusive con el poder de determinar estándares globales en relación con estos nuevos ámbitos de la tecnología. ${ }^{27}$

Por lo tanto, la tarea pendiente tanto de los encargados de las políticas de transformación productiva como de los responsables de los sistemas de CT\&I es la de afinar instrumentos que permitan precaver y/o reaccionar ante los conflictos que puedan surgir en el contexto de los acuerdos de inversión. Para ello es necesario partir de la claridad de que la recomposición del capital que efectúan las grandes corporaciones se hace con fines de maximizar sus utilidades, dejando de lado otros factores tales como la promoción de la innovación o la transferencia de nuevas tecnologías de punta hacia los países receptores (pues éstos desde el principio han sido reconocidos como escenarios para la reducción de costos mediante la externalización de algunas etapas de la cadena de valor). En estas condiciones, resulta fundamental garantizar el que las autoridades encargadas puedan profundizar en los efectos distributivos inherentes a

aislada o poco probable, las disputas sobre patentes y propiedad intelectual son reconocidas como materia arbitrable. en todos los países de América latina, con excepción de México, BANCO DE DESENVOLVIMENTO DA AMÉRICA LATINA. Regulación de la inversión extranjera en América Latina. Regulaciones y opciones para reformas al clima de inversión. 2014. (Serie Políticas Públicas y Transformación Productiva No14). p .71.

27 En este contexto es pertinente el comentario de GHIDINI, G. Innovation: competition and consumer welfare in intellectual property law. Cheltenham: Edward Elgar, 2010. p. 214, respecto de la importancia de la gestión de DIP's para la innovación incremental. Al respecto anota: "The incremental nature of innovation carries with itself the need for innovators to have free access to the fruit of third parties' efforts in order to preserve the creative process. But when proprietary rights come into play (specially in countries like the United States, where patentability tend to extend even to 'research tools'), inventors need to negotiate several licenses to get feu vert to their innovation: hence if transaction costs happen to be too high the very same innovative process could came to a halt" las políticas de ST\&I, ${ }^{28}$ permitiendo situar las decisiones gubernamentales en esta materia más allá del estimulo de sectores productivos para adentrarse en la solución de problemas sociales puntuales. En últimas, se trata de que la promoción a la ST\&I como función inherente al Estado pueda, llegado el caso, erigirse como argumento claro frente a las eventuales pretensiones excesivas por parte de empresas multinacionales.

\section{Consideraciones finales}

Sin lugar a dudas existe un contrasentido en buscar apalancar el desarrollo científico a partir de las rentas del sector extractivo, las cuales por su carácter volátil, pueden dejar en mala situación la financiación de actividades que requieren capital de alto riesgo y largo plazo como son las vinculadas a la $\mathrm{I}+\mathrm{D}+\mathrm{i}$. El actual desaceleramiento de la economía china y los signos de recuperación de la economía Americana después de la crisis financiera de 2008, han vuelto a ubicar a los países latinoamericanos en una situación de reducción de rentas con base en las cuales se diseñaron sus políticas de CT\&I.

Desde esta perspectiva, es fundamental que, aún en medio de la crisis, los países logren la vinculación de los sistemas de innovación a sectores económicos más estables. Una posibilidad para lograrlo, es el recurso a la creación de un mercado de soluciones técnicas y de desarrollo de tecnologías enfocadas a la solución de los problemas que afectan a la de manera directa a la población de los países en desarrollo. Por esta vía, no sólo se configura un mercado interno - que demanda soluciones - sino que también se asegura la eficiencia social del gasto en materia de CTI (muy cuestionable cuando sólo se busca establecer vínculos con EMN's a través de producción científica de alto rango). Finalmente, asumir tal postura podría lograr la atracción de IED estimulada por la expansión de un mercado local con espacios para la colaboración científica.

En este sentido es necesario que las administraciones nacionales asuman la fatalidad de los ciclos económicos con creatividad, reconociendo que la brecha tecnológica de nuestros países nos ubica en nichos de

28 BOZEMAN, Barry; SLADE, Catherine; HIRSCH, Paul. Inequity in the distribution of science and technology outcomes: a conceptual model. Berlim: Springer Science+Business Media, 2011. 
mercado diferentes, lo cual no implica necesariamente que sean mercados poco atractivos o de bajo nivel técnico; por el contrario, su potencial representa la esperanza no sólo para la estabilidad de los SNCT\&I sino también de los destinatarios de las soluciones técnicas que de allí puedan emanar.

Una posibilidad de futuro para los programas de CT\&I después de la crisis de los commodities dependerá de la capacidad para crear nuevos mercados, y aprovechar los recursos existentes y las capacidades creadas para configurar entornos favorables a emprendedores más que a inversionistas inmersos en los patrones clásicos de la inversión que mejora la productividad pero destruye el empleo o a aquella inversión que promueve economías de enclave y además solo busca protegerse de los cambios regulatorios por la vía del arbitramento internacional de inversiones.

El camino está claro, los cambios a realizar son profundos y requieren el compromiso y la transparencia quienes deben liderarlos, de lo contrario en lugar de una promoción de la inversión orientada hacia la innovación lo que tendremos será un área fecunda para el litigio en el plano del arbitramento internacional de inversiones.

\section{REFERÊNCIAS BIBIOGRÁFICAS}

AROCENA, R; SUTZ , J. Desigualdad: tecnología e innovación en el desarrollo latinoamericano Iberoamericana. Nueva época, 2001.

BOZEMAN, Barry; SLADE, Catherine; HIRSCH, Paul. Inequity in the distribution of science and technology outcomes: a conceptual model. Berlim: Springer Science+Business Media, 2011.

BANCO DE DESENVOLVIMENTO DA AMÉRICA LATINA. Regulación de la inversión extranjera en América Latina: regulaciones y opciones para reformas al clima de inversión. 2014. (Serie Políticas Públicas y Transformación Productiva $\mathrm{N}^{\circ} 14$ )

FEDESARROLLO. 20 años de políticas de competitividad en Colombia. Bogotá. 2013.

GERVAIS, Daniel. Current issues in international intellectual property norm-making. In: DREXL, J. et al. (Ed.) Eu bilateral trade agreements and intellectual property: 3 for better or worse?, MPI Studies on Intellectual Property and Competition Law 20. Berlin: Springer-Verlag, 2014.

GHIDINI, G. Innovation: competition and consumer welfare in intellectual property law. Cheltenham: Edward Elgar, 2010.

LIZUKA; Soete. Catching up in the twenty first century: globalization, knowledege and capacities in latin america: a case for natural resource based activities. [Amsterdã]: Unu_Merit Working Paper Series, 2011.

MAZZUCATO, M. The entrepreneurial state: debunking public vs. private sector myths. Londres: Anthem, 2013.

MEZUE et al. The power of market creation: how innovation can spur development, foreign affairs, Enero de 2015. Available in: <m.foreignaffairs.com/articles/142495/ bryan-c-mezue-clayton-m-christensen-and-derek-vanbever/the-power-of-market-creation>.

MOWERY, D.; SIMCOE, T. The Internet. In: STEIL, B.; VICTOR, David G.; NELSON, R. Technological innovation and economic performance. Princeton: Princeton University, 2002.

RED DE INDICADORES DE CIENCIA Y TECNOLOGÍA IBEROAMERICANA E INTERAMERICANA. Red de indicadores de ciencia y tecnología iberoamericana e interamericana. 2014. Available in: <http://www. ricyt.org $>$.

RODRIK, D. One economics: many récipes. Princeton: Princeton University, 2007. 
Para publicar na Revista de Direito Internacional, acesse o endereço eletrônico www.rdi.uniceub.br ou www.brazilianjournal.org.

Observe as normas de publicação, para facilitar e agilizar o trabalho de edição. 